To appear in Ap. J. Letters

\title{
Anomalous Motion of Ionized Gas in the Sickle (G0.18-0.04) Near the Galactic Center
}

\author{
F. Yusef-Zadeh \\ Department of Physics and Astronomy, Northwestern University, Evanston, Il. 60208 \\ (zadeh@ossenu.astro.nwu.edu) \\ D. A. Roberts \\ NCSA, 405 N. Mathews Ave, Urbana, IL 61801 (droberts@lai.ncsa.uiuc.edu) \\ M. Wardle \\ Special Research Centre for Theoretical Astrophysics, University of Sydney, NSW 2006, Australia \\ (wardle@physics.usyd.edu.au)
}

\begin{abstract}
We present VLA measurements of $\mathrm{H} 92 \alpha$ radio recombination line emission from an unusual HII region G0.18-0.04, the "Sickle", with spatial and spectral resolutions of $277^{\prime \prime} 8 \times 24^{\prime \prime} .9$ and $14 \mathrm{kms}^{-1}$, respectively. These observations detected two new kinematic components of ionized gas whose velocities differ greatly from the $+25 \mathrm{kms}^{-1}$ molecular cloud surrounding the Sickle. One component is highly red-shifted with peak velocity of about $+150 \mathrm{kms}^{-1}$ and the other is a blue-shifted velocity feature peaking near $-35 \mathrm{kms}^{-1}$. Neither of these high-velocity features have molecular counterparts. The blue-shifted feature is forbidden in the sense of Galactic rotation and coincides with the prominent nonthermal filaments crossing the Sickle, thus suggesting that they are physically associated with each other. The results presented here are interpreted in terms of ionized gas being accelerated away from the surface of the cloud associated with the Sickle region, either by the magnetic field associated with the nonthermal filaments or by the stellar winds from the hot helium stars near G0.18-0.04.
\end{abstract}

Subject headings: galaxies: ISM - Galaxy: center - ISM: individual (Sgr A East and Sgr A West) — ISM: magnetic fields 


\section{Introduction}

The unusual source G0.18-0.04, the "Sickle", is where the nonthermal filaments (NTFs) of the Galactic center Arc appear to coincide with a thermal source at the Galactic plane (Yusef-Zadeh, Morris \& Chance 1984; Yusef-Zadeh \& Morris 1987a). The filaments are long, narrow synchrotron-emitting features tracing organized magnetic fields which run perpendicular to the Galactic plane (Yusef-Zadeh \& Morris 1987b). G0.18-0.04 is one of the most interesting regions in the Galactic center because of its potential to provide important clues as to the nature of the acceleration of relativistic particles of the NTFs and on the ionization mechanism of thermal gas.

Radio recombination and molecular line studies of G0.18-0.04 detected ionized thermal gas with a Sickle-shaped appearance at a velocity near $+40 \mathrm{kms}^{-1}$ delineating the eastern edge of the $+25 \mathrm{kms}^{-1}$ molecular cloud (Yusef-Zadeh, Morris, \& van Gorkom 1989; Serabyn \& Güsten 1991). G0.18-0.04 is thought to be photoionized by a number of hot helium stars that have been discovered nearby (Moneti, Glass \& Moorwood 1991; Figer, McLean \& Morris 1995; Cotera et al 1996), and the infrared and radio recombination line ratios are consistent with this picture (Harris et al 1994; Timmermann et al 1996; Lang, Goss \& Wood 1997; Simpson et al. 1997)

Interferometric CS observations by Serabyn \& Morris (1994) have shown clumps of molecular gas aligned along the NTFs, supporting the suggestion that the NTFs arise through an interaction with a Galactic Center molecular cloud with the strong, ambient magnetic field believed to permeate the region (Serabyn \& Güsten 1991). In this scenario, the interaction between the magnetic field and the ionized gas at the cloud surface loads energetic particles onto the field lines and the resultant synchrotron emission produces the NTF.

In this Letter we present low spatial and spectral resolution $\left(26^{\prime \prime}\right.$ and $\left.14 \mathrm{kms}^{-1}\right)$ observations of G0.18-0.04. The observations complement the detailed high-resolution $\left(6^{\prime \prime}\right.$ and $\left.8.5 \mathrm{kms}^{-1}\right)$ H92 $\alpha$ observations of Lang et al (1997) by their increased sensitivity to low surface brightness emission. We report the detection of two extreme low- and high-velocity ionized features in the Sickle at $V_{L S R}=-35$ and $+150 \mathrm{kms}^{-1}$. The low-velocity features are associated with the sites of interaction of the NTFs with the Sickle, and further strengthen the connection between the Sickle and the NTFs.

\section{Observations}

H92 $\alpha$ observations of the Sickle were carried out on 14 July 1988 using the D-configuration of the Very Large Array of the National Radio Astronomy Observatory円. A preliminary account

\footnotetext{
${ }^{1}$ The National Radio Astronomy Observatory is a facility of the National Science Foundation, operated under a cooperative agreement by Associated Universities, Inc.
} 
of this observation was described by Yusef-Zadeh et al (1989). This observation was centered at $\alpha(1950)=17^{\mathrm{h}} 43^{\mathrm{m}} 05^{\mathrm{s}}, \delta(1950)=-28^{\circ} 48^{\prime} 45^{\prime \prime}$.

NRAO 530 and 3C 48 were used as phase and flux density calibrators. Bandpass solutions were obtained using both a one hour observation of 3C 84 and the periodic observations of the complex gain calibrator, NRAO 530. The solutions obtained using NRAO 530 were superior to those obtained using $3 \mathrm{C} 84$, due to the fact that they were determined periodically (every 30 min) in time and could track the short time scale variation in the bandpass; thus, the bandpass was corrected using NRAO 530. The correlator was set to observe 32 channels in right circular polarization with a total bandwidth of $12.5 \mathrm{MHz}$ centered on $V_{L S R}=+60 \mathrm{kms}^{-1}$. After on-line Hanning smoothing, the data covered a velocity range between $-162<V_{L S R}<+275 \mathrm{kms}^{-1}$ with a channel resolution and separation of $14 \mathrm{kms}^{-1}$. After careful editing of short-spacing visibilities, standard calibration was carried out. In order to emphasize the weak, extended structures, the visibility data were naturally weighted and tapered at $5 \mathrm{k} \lambda$ giving an angular resolution of $27^{\prime \prime} .8$ $\times 24^{\prime \prime} .9\left(\mathrm{PA}=61^{\circ}\right)$. The continuum channels were fitted and subtracted in the visibility domain using UVLIN in AIPS; the resulting continuum-subtracted data set was imaged. The continuum image was formed by averaging the visibility data in the line-free channels. The typical rms noises for a single line channel and for the continuum are $\approx 0.55$ and $1.58 \mathrm{mJy} \mathrm{beam}^{-1}$, respectively. The negative features near bright sources in the final images are a result of structure in spatial frequencies smaller than those sampled in these observations. The images of integrated line emission and velocity fields were created with the MOMENT program in the MIRIAD software package of the Berkeley-Illinois-Maryland-Association (BIMA). During the moment analysis, the line intensity was used only where the emission was above $2 \mathrm{mJy}^{\text {beam }}{ }^{-1}$ (signal-to-noise ratio $[\mathrm{S} / \mathrm{N}] \approx 4)$.

\section{Results}

The bottom right panel of Figure 1 shows gray scale and contour representations of the continuum emission from the inner $20 \mathrm{pc}$ of the Sickle feature. The continuum contours show the diagonal SE-NW linear feature running perpendicular to the Galactic plane and crossing the Sickle. In high-resolution observations (Yusef-Zadeh \& Morris 1987a,b), the linear feature is resolved into a system of narrow and long NTFs. This low-resolution continuum image shows clearly that the system of linear filaments become rather discontinuous and weaker in surface brightness as they cross the Galactic plane and extend to the northwest of the Sickle. The continuum emission associated with the linear feature peaks at a flux density of $\approx 150$ mJy near $\alpha(1950)=17^{\mathrm{h}} 43^{\mathrm{m}} 10^{\mathrm{s}}, \delta(1950)=-28^{\circ} 49^{\prime}$. In high-resolution images, this peak feature appears to be extended to the northern half of the Sickle; this feature is called the "Wake" in the schematic diagram of Yusef-Zadeh \& Morris (1987a). The circular-shaped source G0.15-0.05, the "Pistol", is the brightest continuum feature in Fig. 1 located near $\alpha(1950)=17^{\mathrm{h}} 43^{\mathrm{m}} 05^{\mathrm{s}}, \delta(1950)=-28^{\circ} 49^{\prime}$. A north-south feature near the southern half of the 
Sickle at $\alpha(1950)=17^{\mathrm{h}} 42^{\mathrm{m}} 55^{\mathrm{s}}, \delta(1950)=-28^{\circ} 50^{\prime} 02^{\prime \prime}$ in extent is also noted. The three panels surrounding the continuum image of Fig. 1 show three spectra taken toward positions marked as crosses on the continuum image.

The most interesting result is the detection of extended blue-shifted ionized gas which is forbidden in the sense of the Galactic rotation. Figure 2 shows a gray-scale image of the H92 $\alpha$ line emission, integrated between -63 and $-7 \mathrm{kms}^{-1}$, overlayed with contours of continuum emission. A typical spectrum of this new feature is shown in position 1 of Fig. 1 with a $\mathrm{S} / \mathrm{N}$ of $\approx 7.6$ and a peak flux density of $4.2 \mathrm{mJy}$ at $\approx-36.0 \mathrm{kms}^{-1}$. Most of the blue-shifted velocity feature is distributed in the diffuse region to the east of the Sickle and to the north of the nonthermal linear feature.

The integrated line emission peaks at $\alpha(1950)=17^{\mathrm{h}} 43^{\mathrm{m}} 3.9, \delta(1950)=-28^{\circ} 47^{\prime} 55^{\prime \prime}$, coincident with the location of one of the NTFs having the continuum flux density of $83.5 \mathrm{mJy}^{\mathrm{beam}^{-1}}$ as it crosses the Sickle. The brightness of the continuum emission from the diagonal linear feature becomes rather weak exactly where the blue-shifted ionized feature peaks. High-resolution radio continuum images of this area are dominated by the narrow, nonthermal filaments (Yusef-Zadeh \& Morris 1987a). Assuming that the emitting gas for this particular component is in LTE with the line-to-continuum ratio of $5 \%$ and an electron temperature of $9800 \mathrm{~K}$ (see below), the total ionized mass and the average electron density for the negative velocity feature are estimated to be $\approx 30 M_{\odot}$ and $150 \mathrm{~cm}^{-3}$, respectively. These values are based upon assumed model geometries and are uncertain by a factor of three.

The other peaks in integrated emission at $\alpha(1950)=17^{\mathrm{h}} 43^{\mathrm{m}} 3.5, \delta(1950)=-28^{\circ} 46^{\prime} 45^{\prime \prime}$ and $\alpha(1950)=17^{\mathrm{h}} 42^{\mathrm{m}} 58^{\mathrm{s}}, \delta(1950)=-28^{\circ} 48^{\prime} 15^{\prime \prime}$ are generally consistent with the high-resolution observations of Lang et al (1997), who noted blue-shifted velocity features in the region where the $-35 \mathrm{kms}^{-1}$ feature crosses the northern half of the Sickle (see their L2 and L3 spectra) and at the southern tip of the Sickle (their L8 line profiles). Blue-shifted emission is present in the [NeII] 12.8 $\mu \mathrm{m}$ spectrum taken toward the Sickle (see Fig 6 of Serabyn \& Güsten 1991), and possibly in the [OIII] $88 \mu \mathrm{m}$ spectrum taken by Timmermann et al (1996) towards the southernmost peak of the integrated line emission in Fig. 2 (see their Fig. 3a).

Figure 3 is a gray-scale representation of the highest red-shifted velocity features between +106 and $+205 \mathrm{kms}^{-1}$ with continuum emission contours superposed. Two highly red-shifted components are noted in this figure. One is the velocity feature associated with the Pistol located to the south of the linear feature having a peak velocity of $+125 \mathrm{kms}^{-1}$ (Yusef-Zadeh et al 1989; Lang et al 1997). The second component is a new high-velocity redshifted feature distributed close to the peak continuum emission from the linear feature. This feature has a peak velocity of $\approx 150 \mathrm{kms}^{-1}$ at $\alpha(1950)=17^{\mathrm{h}} 43^{\mathrm{m}} 10^{\mathrm{s}} \cdot 3, \delta(1950)=-28^{\circ} 48^{\prime} 27^{\prime \prime}$. Position 2 of Fig. 1 presents the spectrum of the peak line emission with a flux density of $5.7 \mathrm{mJy}^{\mathrm{beam}}{ }^{-1}$ and a corresponding continuum flux density of $100 \mathrm{mJy}_{\text {beam }}{ }^{-1}$. This newly-detected ionized feature has the highest radial velocity in the Galactic center region with the exception of Sgr A West. The total ionized 
mass and the average electron density for this velocity component are estimated to be $\approx 12 M_{\odot}$ and $170 \mathrm{~cm}^{-3}$, respectively.

Figure 4 shows the velocity distribution of ionized gas ranging between -100 and +190 with contours of total intensity superposed. Note that the extent of high-velocity red-shifted gas beyond $+90 \mathrm{kms}^{-1}$ is not limited to the Pistol but also to the region to the southern half of the Sickle. The new velocity feature peaks at $\alpha(1950)=17^{\mathrm{h}} 42^{\mathrm{m}} 55.3^{\mathrm{s}}, \delta(1950)=-28^{\circ} 49^{\prime} 33^{\prime \prime}$. The spectrum of this $+92 \mathrm{kms}^{-1}$ velocity feature with the peak line emission of $7 \mathrm{mJy}$ is observed in Position 3 of Fig. 1. The total ionized mass and electron density are estimated to be similar to the +150 $\mathrm{kms}^{-1}$ velocity feature.

An accurate estimate of the thermal continuum emission from these diffuse ionized features is quite difficult to make in the presence of the non-thermal emission from the NTFs. However, if the ionized gas is assumed to be LTE and that the abundance of singly ionized helium relative to singly ionized hydrogen $\left(\mathrm{Y}^{+}\right)$is $10 \%$, the upper limits to the electron temperature can be estimated. For positions 1, 2, and 3 (see Fig. 1) upper limits to the electron temperatures are estimated to be 9800,6100 , and $2900 \mathrm{~K}$, respectively. The largest uncertainty from these estimates comes from the fact that the thermal continuum flux cannot be distinguished from nonthermal continuum. Unlike positions 1 and 2, which are near NTFs, position 3 (the southern half of the Sickle) does not appear to be contaminated by any emission from NTFs, thus the estimated electron temperature at this position is not an upper limit, but rather is probably the actual value. This low value of electron temperature is less than that of ionized gas ranging between $4600 \mathrm{~K}$ and $7000 \mathrm{~K}$ in Sgr A West (Roberts and Goss 1993; Yusef-Zadeh, Zhao and Goss 1995). It should be stated that because of its weak surface brightness and its location, the determination of the electron temperature of this particular velocity component may suffer from the lack of short-spacing data.

\section{Discussion}

The peaks of blue-shifted H92 $\alpha$ emission lie exactly on the three bundles of filaments that intersect the Sickle, supporting the notion that there is an interaction between the NTFs and the thermal ionized gas in the Sickle. The results of the observations are generally consistent with the idea that cloud material ionized by UV radiation from hot stars is accelerated from the surface

and some of the gas becomes attached to the magnetic filaments and is accelerated to relativistic velocities where it emits synchrotron radiation (Serabyn \& Morris 1994; Timmermann et al 1996). Indeed, the synchrotron-emitting vertical filaments crossing the Sickle show an intrinsic positive spectral index (Yusef-Zadeh 1989; Anantharamaiah et al 1991; Tsuboi et al 1996), which becomes flat or negative toward more negative latitudes away from the Sickle (Pohl, Reich \& Schlickeiser 1992), whereas another group of non-thermal filamentary structures (the "threads") in the Galactic center region shows steep spectral indices but they are relatively isolated and do not coincide with any ionized thermal features (Anantharamaiah et al 1991; Gray et al 1994). The 
flat spectrum of the Arc may indicate that thermal gas associated with the Sickle is mixed with synchrotron emitting nonthermal gas associated with the linear filaments (Yusef-Zadeh \& Morris 1987a; Anantharamaiah et al 1991).

Lang et al (1997) and Timmermann et al (1996) found a velocity gradient across the Sickle in a direction parallel to the filaments, with the largest (redshifted) velocities towards the west, and the smallest (roughly $20 \mathrm{kms}^{-1}$ ) to the east. The blue shifted gas generally lies to the east of the Sickle, consistent with this velocity gradient. The densest ionized gas is dynamically coupled to the $+25 \mathrm{kms}^{-1}$ molecular cloud whereas the low-density diffuse features presumably originated at the outer surface of the cloud where they were accelerated to anomalous velocities. Lang et al (1997) find an unusually high H115 $\beta / \mathrm{H} 92 \alpha$ ratio, inconsistent with LTE within the Sickle where the NTFs intersect the Sickle. The infrared observations of Simpson et al (1997) show that the extinction towards the Sickle region is uniform, implying that the Sickle itself lies on the front of the $+25 \mathrm{kms}$ cloud, and therefore that the ionised gas is being accelerated towards us from the surface of the cloud. If the blue-shifted gas is being accelerated along the magnetic field aligned with the NTFs, then some of the filaments must be tilted so that the southeast points toward us.

If the magnetic field is responsible for the acceleration, the magnetic pressure must dominate the ram pressure of the blue-shifted gas. Adopting an electron density of $\approx 150 \mathrm{~cm}^{-3}$ for the -35 $\mathrm{kms}^{-1}$ feature at position 1 , a field strength of at least $0.2 \mathrm{mG}$ is required.

Alternatively, the ram pressure from the combined winds of the hot mass-losing stars discovered near the Sickle (Moneti et al 1991; Figer et al 1995, 1996; Cotera et al 1996) may be sufficient to accelerate the gas. The stars' broad He I and $\operatorname{Br} \gamma$ emission lines indicate terminal velocities in the range $V_{w}=500-700 \mathrm{~km} \mathrm{~s}^{-1}$, similar to the cluster of young stars at the Galactic center (Krabbe et al 1991). Adopting a distance of 5 pc from the cluster to the Sickle, the required mass loss from hot stars with $V_{w}=700 \mathrm{~km} \mathrm{~s}^{-1}$ to accelerate the $30 M_{\odot}$ of ionised gas responsible for the feature at peak 1 from the surface of the Sickle at $+25 \mathrm{kms}^{-1}$ to a velocity of $-35 \mathrm{kms}^{-1}$ over a distance of $2 \mathrm{pc}$ is $\approx 3 \times 10^{-3} M_{\odot} \mathrm{yr}^{-1}$.

A ram pressure of this magnitude is also capable of accelerating the redshifted gas, for which the the physical relationship with the NTFs is less clear. The gas at position 2 lies well away from the Sickle HII region, between the two northernmost bundles of filaments that pass through the Sickle. A small continuum feature is present at this position in the $5 \mathrm{GHz}$ image of Yusef-Zadeh \& Morris (1987a) and the $8.3 \mathrm{GHz}$ image of Lang et al (1997). The southeastern end of the Sickle (near position 3) also does not appear to be asociated with any NTFs. The kinetic energy of the features are roughly $3 \times 10^{48}$ and $10^{48}$ erg respectively.

Measurements of the field strength and the total stellar mass loss rate in this region would be useful in discriminating between these mechanisms for accelerating the anomalous-moving clouds.

F. Yusef-Zadeh's work was supported in part by NASA grant NAGW-2518. D. Roberts acknowledges support from the NSF grant AST94-19227. The SRCfTA is funded by the Australian 
Research Council under the Special Research Centres programme.

\section{REFERENCES}

Anantharamaiah, K.R., Pedlar, A., Ekers, R.D., \& Goss, W.M. 1992, MNRAS, 249, 262

Cotera, A.S., Erickson, E.F., Cogan, S.W.J., Simpson, J.P., Allen, D.A., \& Burton, M.J. 1996, ApJ, 461, 750

Figer, D.F., Mc Lean, I.S., \& Morris, M. 1995, ApJ, 447, L29

Figer, D.F., Morris, M. \& Mc Lean, I.S. 1996, The Galactic Center: ASP Conference Series, vol. 102, ed. R. Gredel, p263

Harris, A.I., Krenz,T., Genzel, R., Krabbe, A. \& Lutz, D. 1994, The Nuclei of Normal Galaxies: Lessons from the Galactic Center, ed. R. Genzel \& A. Harris, (Dordrecht:Kluwer), 233

Krabbe, A., Genzel, R., Drapatz, S. \& Rotaciuc, V. 1991 ApJ, 382, L19

Lang, C.C., Goss, W.M. \& Wood, D.O.S. 1997, ApJ, 474, 275

Moneti, A., Glass, I.S. \& Moorwood, A.F.M. 1991, Mem. Soc. Astr. Ital., 62 , 755.

Morris, M. \& Yusef-Zadeh, F. 1989, ApJ, 343, 703

Pohl, M., Reich, W. \&Schlickeiser, R. 1992, A\&A, 262, 441

Roberts, D.A. \& Goss, W.M. 1993, ApJS, 86, 133

Serabyn, E.\& Morris, M. 1994, ApJ, 424, L91

Serabyn, E.\& Güsten, R. 1991, A\&A, 184, 133

Simpson, J. P., Colgan, S. W. J., Cotera, A. S., Erickson, E. F., Haas, M. R., Morris, M. \& Rubin, R. H. 1997, ApJ, 487, in press

Timmermann, R., Genzel, R., Poglitsch, A., Lutz, D., Madden, S.C., Nikola, T., Geis, N. \& Townes, C.H. 1996, ApJ, 466, 242

Tsuboi, M., Inoue, M., Handa, T., Tabara, H., Kato, T. Sofue, Y. and Kaifu, N. 1986, AJ, 92, 818

Yusef-Zadeh, F. 1989, IAU Symposium 136, The Center of the Galaxy, ed. M. Morris (Dordrecht:Kluwer), 275

Yusef-Zadeh, F. \& Morris, M. 1987a, AJ, 94, 1178

Yusef-Zadeh, F. \& Morris, M. 1987b, ApJ, 322, 721

Yusef-Zadeh, F., Morris, M. \& Chance, D. 1984, Nature, 310, 557

Yusef-Zadeh, F., Morris, M. \& van Gorkom, J.H., 1987, The Galactic Center, ed. D.C. Backer (AIP:New York), 190

Yusef-Zadeh, F., Morris, M. \& van Gorkom, J.H. 1989, IAU Symposium 136, The Center of the Galaxy, ed. M. Morris (Dordrecht:Kluwer), 275 
Yusef-Zadeh, F., Zhao, J.-H. \& Goss, W.M. 1995, ApJ, 442, 646

\section{Figure Captions}

Fig. 1. - The gray scale image in the bottom right corner shows the $8.3 \mathrm{GHz}$ continuum emission from the Sickle region with a spatial resolution of $27^{\prime \prime} .8 \times 24^{\prime \prime} .9\left(\mathrm{PA}=61^{\circ}\right)$. Contour levels for this figure as well as Figs. 2, 3, and 4 are represented at 20, 80, 140, 200, and 260 mJy beam ${ }^{-1}$. The three H92 $\alpha$ line profiles are obtained at the positions marked in the continuum image. In the profiles, the crosses show the observed spectra, the solid lines show the model fits, and the dotted lines show the residuals.

Fig. 2. - The H92 $\alpha$ line intensity integrated between -63 and $-7 \mathrm{kms}^{-1}$ is represented as gray scale with superposed contours of continuum emission at $8.3 \mathrm{GHz}$. The peak emission from the blue-shifted velocity feature coincides at the location where the NTFs cross the Sickle.

Fig. 3. - The H92 $\alpha$ line intensity integrated between +106 and $+205 \mathrm{kms}^{-1}$ is represented as gray scale with superposed contours of continuum emission at $8.3 \mathrm{GHz}$.

Fig. 4. - A pseudo-color representation of the velocity distribution of ionized gas covering the entire range between -100 and $+190 \mathrm{kms}^{-1}$. The overlaid contours show the $8.3 \mathrm{GHz}$ continuum emission in the Sickle region. 

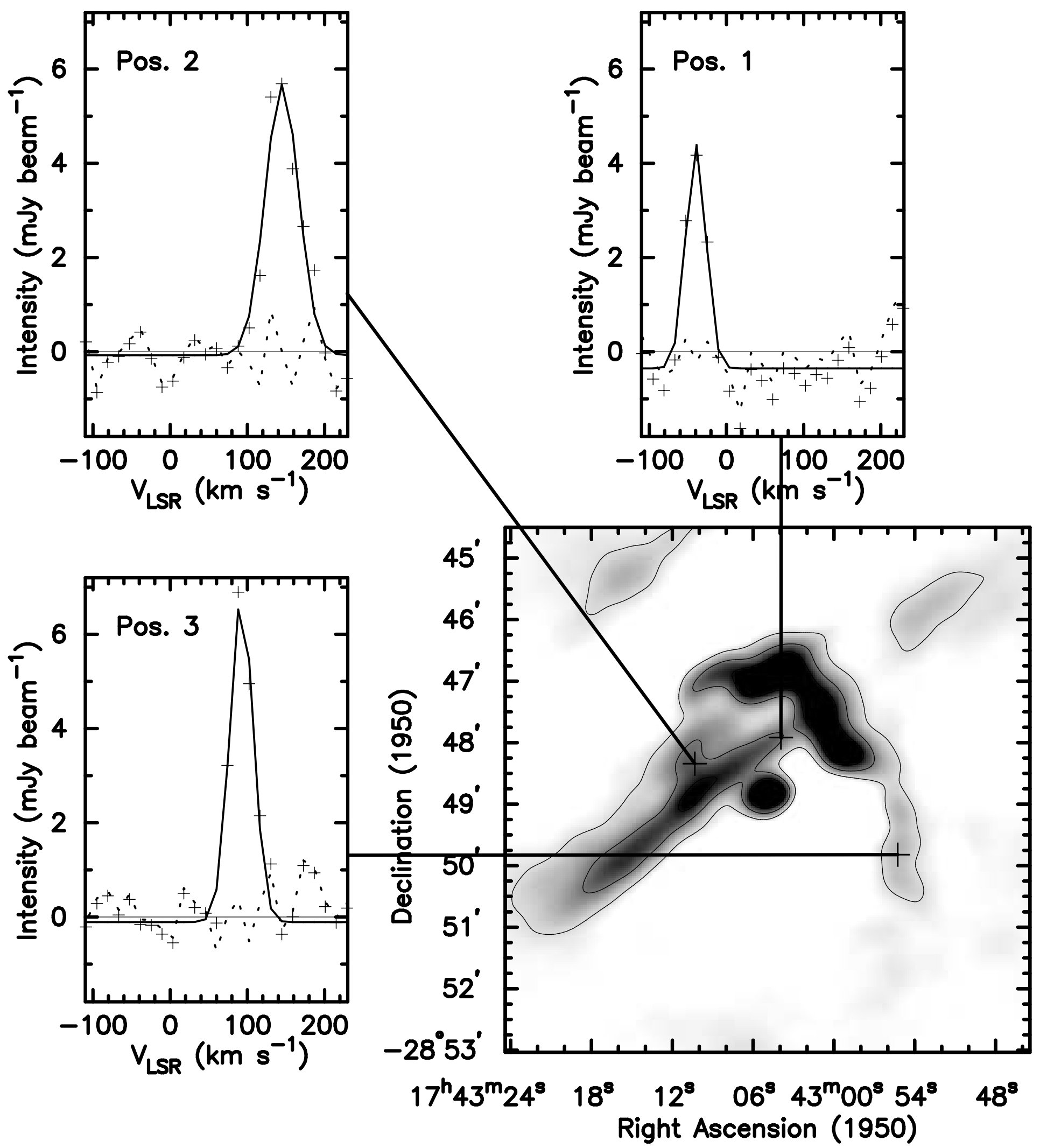


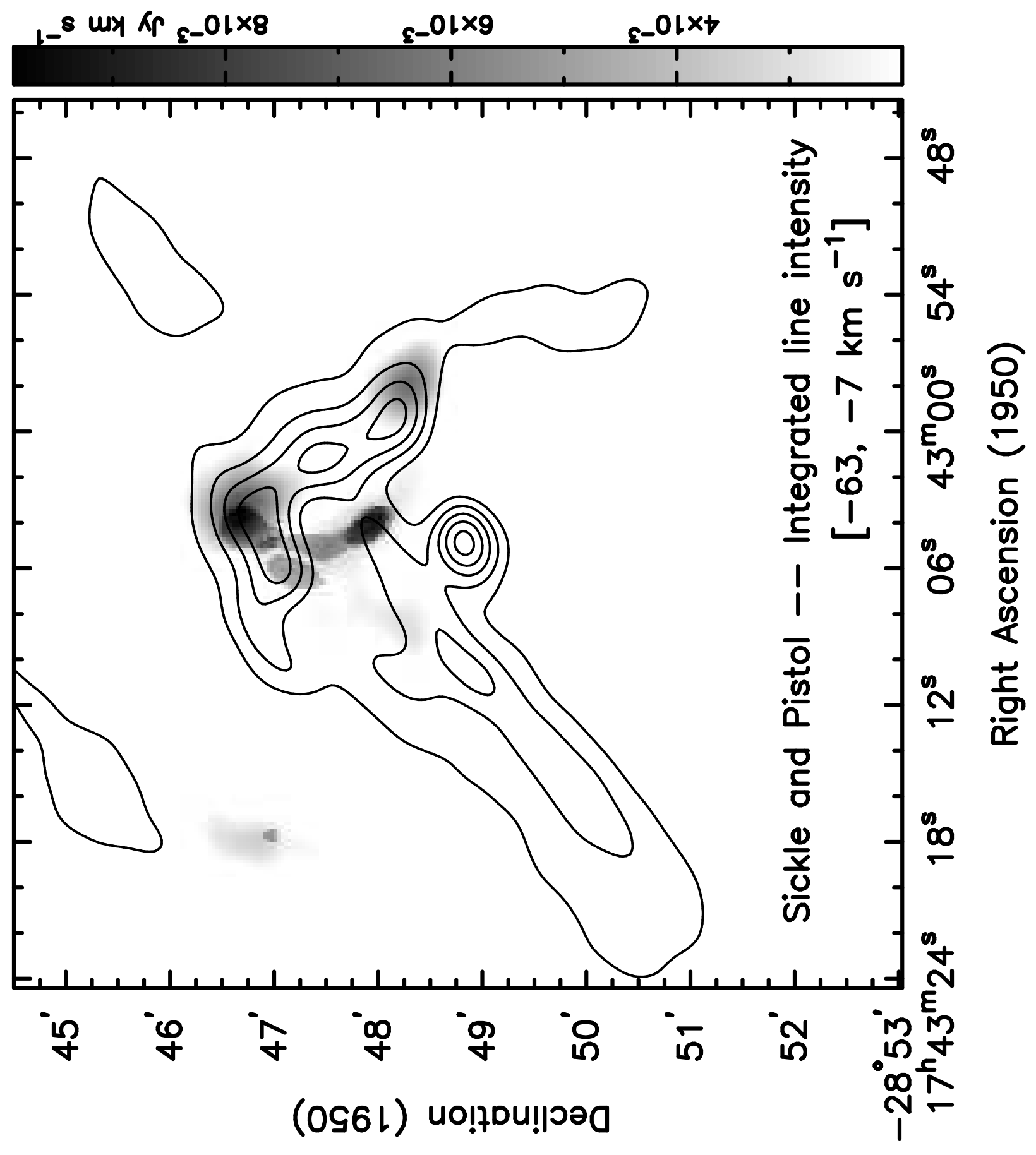




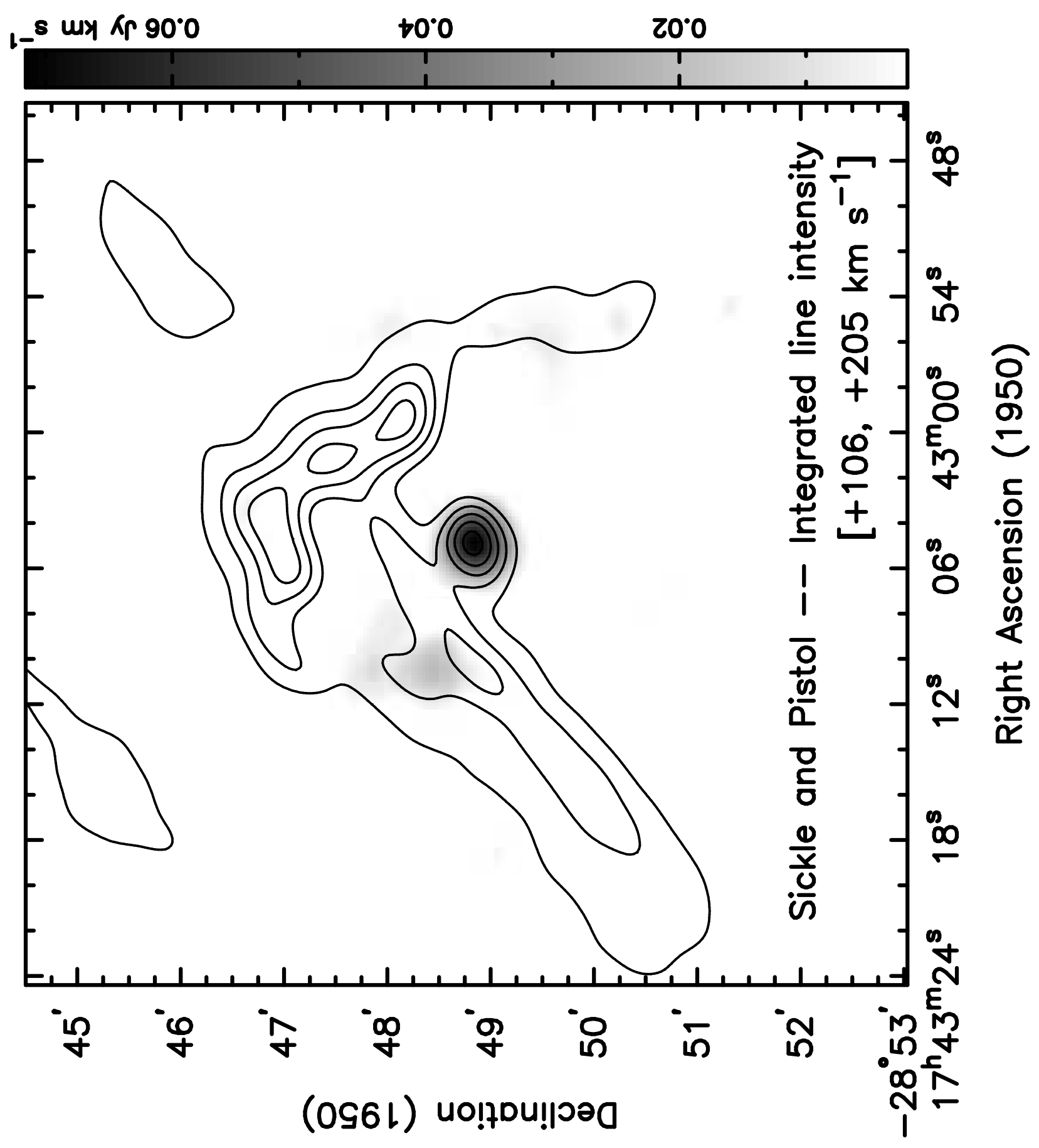


This figure "figure4.gif" is available in "gif" format from: http://arxiv.org/ps/astro-ph/9710046v1 